\title{
Der Schweizer Weltatlas - zur Ausgabe 1981 des Schulatlasses
}

\section{Einleitung}

Seit mehreren Monaten sind sämtliche Ausgaben des Schweizerischen Mittelschulatlasses und des Schweizerischen Sekundarschulatlasses vergriffen. An ihrer Stelle wird seit Frühjahr 1982 von der Konferenz der Kantonalen Erziehungsdirektoren ein Schulatlas mit dem neuen Namen "SCHWEIZER WELTATIAS" herausgegeben. Ueber diese grundlegende Veränderung soll an dieser Stelle durch den neuen Chefredaktor etwas ausführlicher orientiert werden. Nach einem kurzen Rückblick wird die organisatorische Struktur berührt, das Grobkonzept erläutert, das der in Angriff genammen Umarbeitung zugrunde liegt, und die neuen Kartentypen werden kurz vorgestellt und kammentiert.

\section{Vorgeschichte und Organisatorisches}

Seit 1927 betreute Prof. Dr. h.c. Eduard Imhof als Chefredaktor im Auftrag der Konferenz der Kantonalen Erziehungsdirektoren den Schweizerischen Mittelschulatlas. Mit berechtigtem Stolz wird dieses Werk gelegentlich als das erste realisierte Schulkoordinationsprojekt aller Kantone bezeichnet. Diese jahrzehntelange Zusammenarbeit duirfte auf der Einsicht beruhen, dass der beträchtliche Aufwand für ein solches Lehrmittel nur dann einigermassen im Rahmen gehalten werden kann, wenn die Mittel nicht zersplittert werden. Unter der Leitung von Professor Imhof enstanden von 1927 bis 197612 Auflagen in deutscher, 10 in französischer und 8 Auflagen in italienischer Sprache.

In enger Anlehnung an dieses Atlaswerk wurden von ihm in den Jahren 1928 bis 1975 parallel 12 Ausgaben des im Umfang wesentlich dünneren Schweizerischen Sekundarschulatlasses herausgebracht. Als er nach beihnahe 50-jähriger Tätigkeit als Chefredaktor von diesem Amt zurücktrat, durfte er ohne Zweifel auf eine einzigartige Leistung zurückblicken, hatte ihm doch auch die graphische Qualität seiner Karten hohe Anerkennung eingetragen.

Die Atlasdelegation der EDK hatte mit dem Schreibenden bereits Verhandlungen über die Uebernahme der Redaktion aufgenammen, als im Oktober 1975 der Leitende Ausschuss der Interkantonalen Lehrmittelzentrale eine Konzeptkamission Atlas einsetzte, mit dem Auftrag zu prüfen, ob eine Umarbeitung der Schulatlanten notwendig sei. Im Einverständnis mit der Atlasdelegation beteilig- te ich mich an den unfangreichen Arbeiten dieser Konmission, in der im weiteren 4 Vertreter der Geographie der Sekundarschulstufe, 3 der Gymnasialstufe und 1 Vertreter der Hochschulen mitwirkten. Im folgenden wurden Umfragen bei den Benützern durchgeführt, 20 ausländische Atlanten evaluiert und Grundlagen für ein neues Konzept zusammengetragen. Im Dezember 1976 konnte der Schlussbericht der Konzeptkommission abgeschlossen werden. Er wurde im Laufe des Jahres 1977

den Kantonen und weiteren interessierten Stellen zur Vernehmlassung unterbreitet. Im wesentlichen schlug die Kommission eine vollständige Erneuerung und Zusammenfassung zu einem einzigen Atlas vor. Auf die wichtigsten Punkte der Neukonzeption wird im nächsten Abschnitt eingetreten. Sie fand in der Vernehmlassung, von vereinzelten Bedenken abgesehen, eine sehr positive Aufnahme, so dass die EDK, nach eingehender Abklärung der finanziellen Aspekte, im Dezember 1979 auf Antrag der Atlasdelegation dem Projekt für eine Erneuerung des Schulatlasses zustimte. Da die Vorräte aus den bisherigen Auflagen rasch zur Neige gingen, musste man sich für eine Zwischenauflage entscheiden, die möglichst bald publiziert werden konnte. Im Februar 1980 wurden die entsprechenden Verträge unterzeichnet. 2 Jahre später gingen die letzten Bogen in den Druck. Für die Erstellung der Entwürfe der neuen Karten wurde die Redaktion vorerst durch drei Geographen ergänzt; zwei davon verfügen über langjährige Erfahrung aus dem Mittelschulunterricht. Zudem konnte die Mitarbeit weiterer Fachleute bei der Detailkonzeption gewonnen werden. Ueberdies wurde von der EDK zur Unterstützung der Redaktion eine Beratende Kommission eingesetzt, welche zu Aenderungen am Konzept und zu Kartenproben Stellung nimmt, über Anregungen und Vorschläge von Atlasbenützern berät und bei deren Infonmation mitwirkt. Diese Komission steht unter der Leitung von PD Dr. R. Marr, Basel. Gegenwärtig gehören ihr drei Vertreter der Sekundarschulstufe und sechs Vertreter von Gymnasien und Lehrerseminarien an. Für die Zwischenausgabe konnten insgesamt 56 Karten von Grund auf neu erstellt werden.

Der Chefredaktor besorgte neben seiner Leitungsfunktion fast sämtliche Nachführungs- und Ergänzungsarbeiten für alle 209 Karten, welche aus der letzten Ausgabe des Mittelschulatlas übernonmen wurden. Die Uebersetzungen wurden von sprachlich versierten Fachleuten aus den beiden anderen Sprachgebieten vorgenommen und überprïft. 


\section{Die Hauptpunkte des Grobkonzeptes fuir den neuen Atlas}

In der Konzeptkommision gelangte man, nicht zuletzt auch aus dem Studium von Geographie-Lehrplänen und des Ergebnisses von Umfragen zur Auffassung, dass sich der Geographie-Unterricht in den letzten zwei Jahrzehnten inhaltlich, wie auch methodisch und didaktisch grundlegend verändert hat. Die früher stärker länderkundlich und physisch-geographisch orientierten Lehrinhalte wurden abgelöst durch eine mehr die Einwirkungen der Menschen auf ihren Lebensraum betonende Konzeption des Geographie-Unterrichts, wobei häufiger auch thematische Querverbindungen verfolgt werden. Diesen Veränderungen möchte man im neuen Atlas dadurch entgegenkommen, dass zusätzliche thematische Karten beigegeben werden, ohne dass die physisch-geographische Komponenten deswegen vernachlässigt wird. Um vor allem dem schüler die allgemein-geographische Orientierung zu erleichtern, wird jedoch die regionale Gliederung im Atlas beibehalten. Thematische Problemkreise werden über ein Sachregister und Vermerke mit Seitenzahlen erschlossen.

Besonders eingehend wurde die Gestaltung der Länderkarten und zugehörigen Wirtschaftskarten studiert. Die Kormission entschied sich, jeden grösseren Raum durch ein Kartenpaar, bestehend aus einer topographischen Uebersicht und einer komplexen Wirtschaftskarte im gleichen Massstab, darzustellen. Bei den topographischen Uebersichten soll die Farbe nicht zur Unterstützung von Höhenstufen, sondern zur Differenzierung der wichtigsten Vegetationstypen wie Wald, Kulturland, Steppe, Wiste etc. eingesetzt werden. In den Klimakarten sollen zusätzliche Informationen angeboten werden. Eine ganze Reihe von Themenkreisen sind zur Ergänzung des humangeographischen Bereiches vorgesehen. Auch spezifische Probleme der Entwicklungsländer sollen zur Darstellung karmen.

Der Teil Schweiz wird vollständig erneuert und durch Karten neuer Themen ergänzt werden. Im weiteren ist vorgesehen, Kartenspiegel und Buchformat zu verbreitern und letzteres besser auszunützen. Ferner wird auch wegen des Verzichtes allf die Weiterführung des Sekundarschulatlasses der Gesamtumfang erweitert. Durch die bewusste Mischung von leichter lesbaren und komplexeren Karten soll ein auf verschiedenen Stufen brauchbares Angebot geschaffen werden.

\section{Kommentar zu den neuen Kartentypen in der Zwischenauflage}

Im Rahmen der Zwischenauflage konnte erst eines der vorgesehenen Kartenpaare realisiert werden, nämlich Südamerika im Massstab 1:25 Mio. Die beiden Karten sind nach neuesten Grundlagen neu bearbeitet worden. Das Relief wurde der neuen Situation angepasst. Anstelle der Höhenstufen- farbe tritt eine reichhaltige Palette von Vegetationstönen. Sie gliedern den Kontinent in die hauptsächlichsten Landschaftstypen (AmazonasRegenwald, Llanos, Campos, Chaco, Pampa, Puna, Wistenstreifen der pazifischen Küste etc.). Um die Zahl der nötigen Farbtöne zu reduzieren, wurden verwandte Kategorien durch zusätzliche Strukturraster unterschieden. Relativ dunke Farbtöne wurden den Wäldern zugewiesen, helle Farbtöne den Gebieten mit karger Vegetation. Eine gewisse Entlastung durch Reduktion der Zahl der Vegetationstypen drängt sich noch auf, damit die Reliefwirkung verbessert und die Karte besser lesbar wird. Ein Versuch, noch Höhenlinien miteinzubeziehen, hat sich als graphisch nicht lösbar erwiesen.

Die zugehörige Wirtschaftskarte vereinigt sämtliche wirtschaftlichen Aktivitäten in mehreren über- und ineinandergefügten Schichten. Für die vorwiegende Landnutzung stehen relativ helle Flächentöne zur Verfügung (Abb. 1). Spezialund Nebenkulturen sind mit kräftigen bildhaften Einzelsignaturen aufgesetzt (Abb. 2). Die Gewinnung von Bodenschätzen und Energie wird mit einfachen geometrischen Signaturen und kräftigen Farben betont, ebenso die verschiedenen zugehörigen Verarbeitungsstätten (Abb. 3). Die Städte sind ebenfalls je nach Schwerpunkt der Aktivitäten farblich differenziert (Abb. 4). Die wichtigsten Transportwege der Rohstoffe, Energieträger und verschiedenen Produkte zu Lande und zu Wasser bilden die linearen Elemente der Karte (Abb. 5). Die Basiskarte dazu umfasst das Gewässernetz, die Küstenlinien, die Staatsgrenzen und ein abgeschwächtes Relief (Abb. 6). Die Auswahl der Namen umfasst neben den bedeutendsten Städten auch spezifische, vom Gesichtspunkt der Wirtschaft interessante Namen, z.B. von grösseren Bergbauminen, von ihren zugehörigen Ausfuhrhäfen, von Standorten von Kernkraftwerken etc. Bei der Farbgebung wurde darauf geachtet, dass die eine Branche charakterisierende Farbe über alle Stufen hinweggeht. So ist z.B. das Dunkelrot für Erdöl eingesetzt auf dem Erdölfeld, auf dem Transportweg, für die Raffinierie, für die darauf aufbauende chemische Industrie, wie auch im Diagramm über Ein- und Ausfuhr der einzelnen Länder. Auf diese Weise sollte es möglich sein, einzelne Produkte von ihrer Gewinnung bis zur Verarbeitung zu verfolgen. Der relativ grosse Massstab erleichtert die von vielen Benützern gewïnschte detaillierte Darstellung.

Alle Signaturen für den Bergbau und die Energieproduktion wurden nach 3 Grössenstufen unterschieden. Bei Kohle, Erdgas, Eisenerz und Stahl entspricht die grösste Signatur grössenordnungsmässig 1 \% der Weltproduktion. Bei den übrigen Bodenschätzen umfasst sie ca. 5 \%, die Signatur mittlerer Grösse jeweils die Hälfte. Die kleinste Signatur steht für Vorkamen von geringer Bedeutung. Bei den Seetransporten sind die Bandbreiten proportional zu den jährlich beförderten Tonnagen wiedergegeben.

Mit der gewählten Darstellungsform, dem komplexen Gefüge aus vielen für die Wirtschaftsstruktur wichtigen Aspekten, lassen sich besonders 


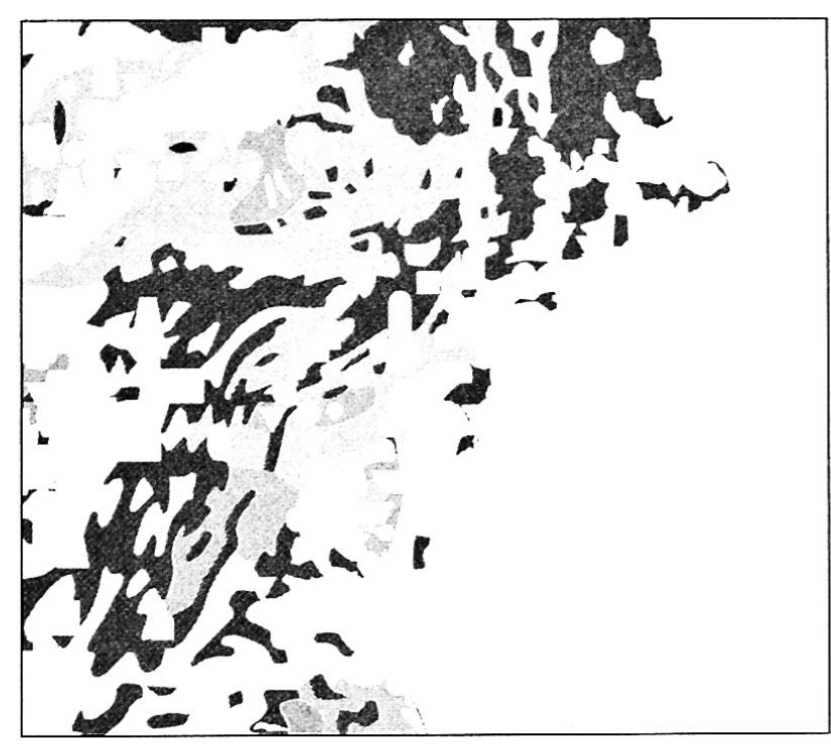

Abb. 1 Auszug aus der Wirtschaftskarte USA, Schicht Landnutzung

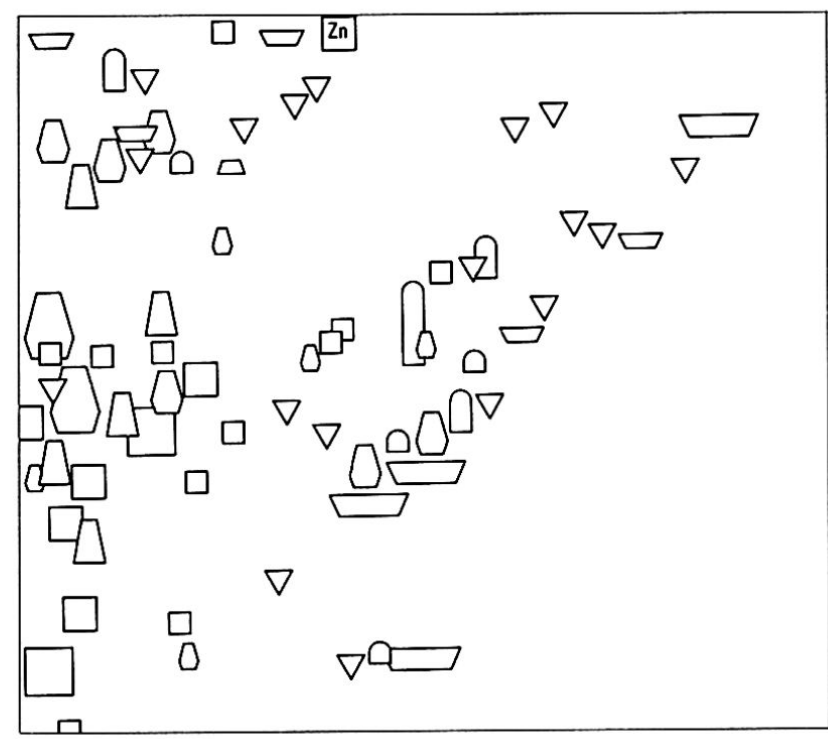

Abb. 3 Schicht Gewinnung von Bodenschätzen und Verarbeitungsstätten

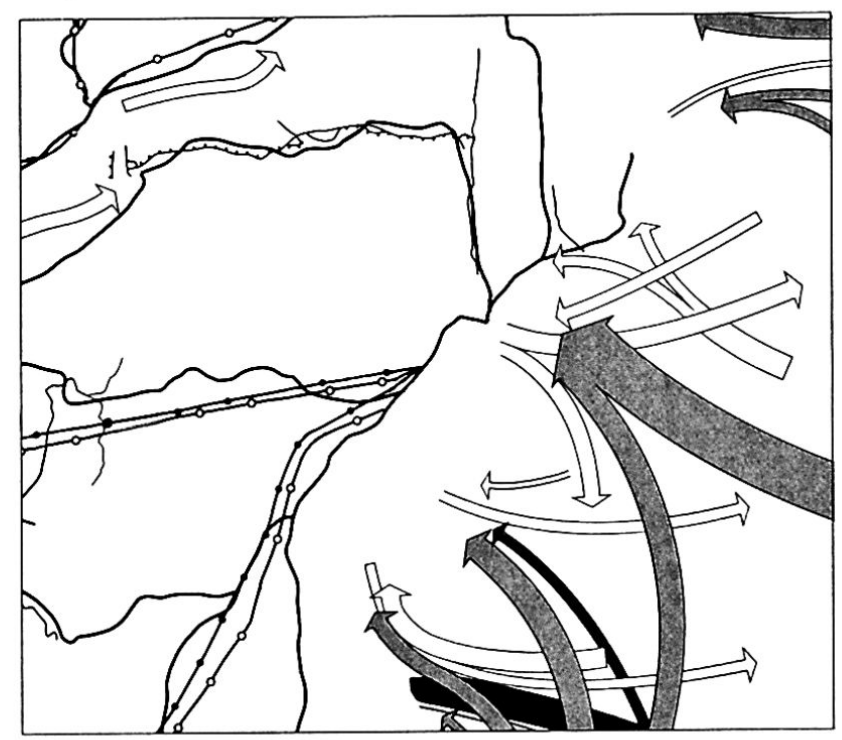

Abb. 5 Schicht Transportwege mit Bahnen, Kanälen, Erdölund Gasleitungen und Schiffstransportrouten

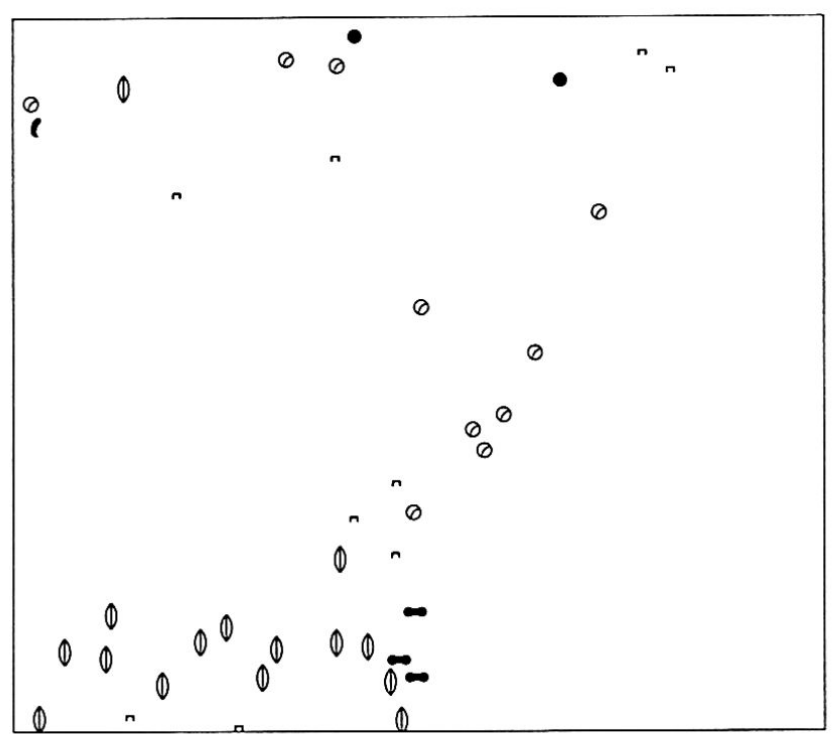

Abb. 2 Schicht Neben- und Spezialkulturen

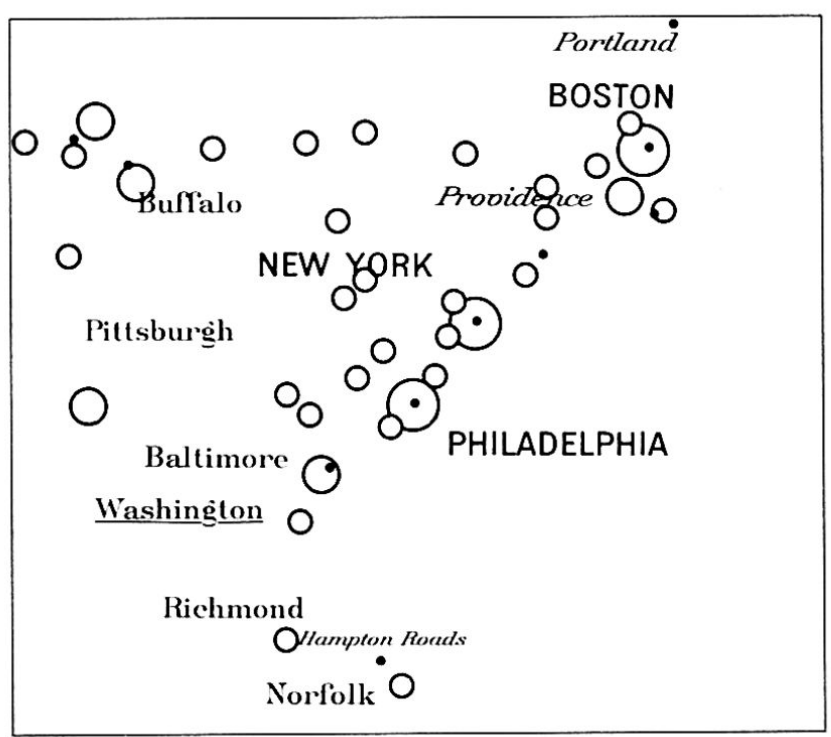

Abb. 4 Schicht Industriestädte und Häfen

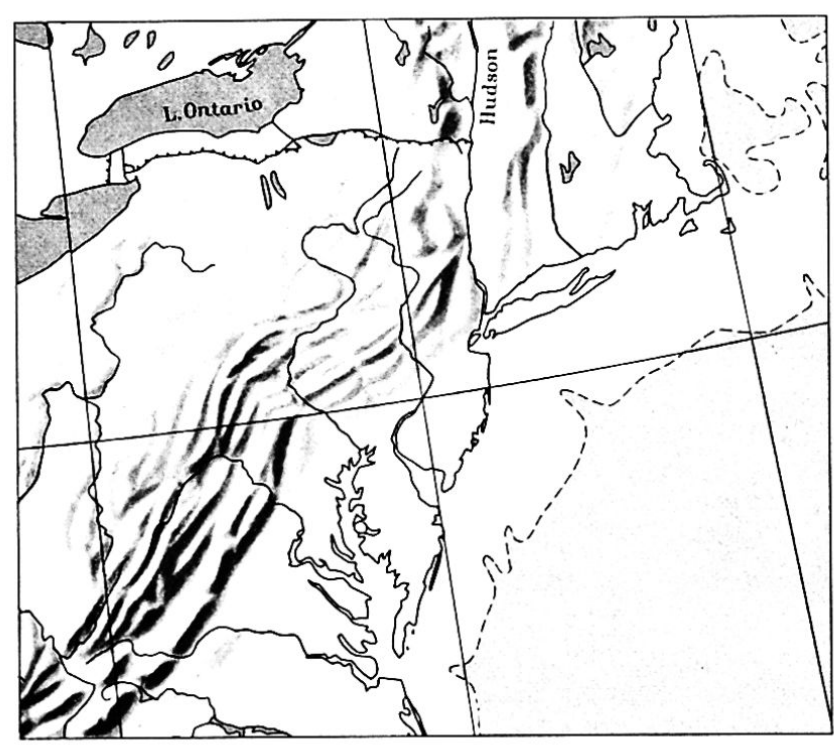

Abb. 6 Basiskarte zur Wirtschaftskarte USA im Schweizer Weltatlas mit Gewässernetz und Relief 
in idealer weise geographische Teilräume analysieren. Das Zusammenwirken der verschiednen Komponenten wird erkennbar. Benachteiligte Räume heben sich ab von begünstigten. Stark mit Industrie belastete Gebiete werden deutlich herausgestellt. In diesen Belangen ist die neue Wirtschaftskarte Kanada-USA-Mexiko (1:15 Mio) der Zwischenauflage 1981 dank des grösseren Massstabes gegenüber der Karte Südamerika im Vorteil. Um die kleinräumigeren Verhältnisse wiedergeben zu können, wurde für die Wirtschaftskarte Mittelamerika (1:10 Mio) eine andere, vereinfachte Flächendarstellung gewählt. Wenn auch auf solche Weise auf die Besonderheiten jedes Raumes Rücksicht genommen werden kann, muss doch eine gewisse Einheitlichkeit beachtet werden, wenn die Karten verschiederner Gebiete der Erde vergleichbar bleiben sollen.

Für die Auflage 1981 wurden auch die Klimakärtchen der europäischen Länder neu entworfen, wobei fast durchwegs neuere Grundlagen benützt werden konnten. Aus der Ueberlegung, dass gewisse Klima-Komponenten stark reliefbedingt sind, wurde dem üblichen Farbstufenaufbau für Monatsmittel der Temperatur und Jahresniederschläge das Relief unterlegt. Es erleichtert zweifellos die Orientierung und macht manchen Sachverhalt einsichtiger. Um allfällige Schwierigkeiten bei der Identifizienung einzelner Farbstufen zu mildern, wurden etwas grössere Stufen als im Mittelschulatlas gewählt. Im Hinblick auf die nächste Auflage wurden diese Klimakarten bereits im Entwurf zu Europakarten zusammengefasst. Einige Hinweise über die vorgesehene Erneuerung zeigen die Seiten 1 - 13 der Zwischenauflage. Alle diese Karten wurden nach dem beschlossenen Konzept neu entworfen und kartographisch bearbeitet. Der Teil Schweiz soll noch um einiges erweitert werden, um die beim heutigen Inhalt vorhandenen Lïcken zu schliessen. Der generelle Trend dürfte aus den bereits vorliegenden Karten dennoch erkennbar sein. Wir können hier nur einige wenige Karten herausgreifen. S. 4 mit der Klus von Moutier ist Teil einer Serie von Einführungskarten ins Kartenlesen. Die farbige Schrägaufnahme aus der Luft und die dazugehörige einfache Karte in Grundrissdarstellung können Detail für Detail miteinander verglichen werden. Die anschliessende Seite bringt einen Querschnitt durch den Tafel- und Kettenjura in Form einer geologischen Karte und von 3 geologischen Profilen, alle im Massstab 1:100 000. Dieses Material dürfte nützlich sein als Einführung in fundamentale Sachverhalte der Geologie. Trotz teilweise recht komplizierten Verhältnissen werden stratigraphische und tektonische Erscheinungen fast modellhaft sichtbar. Im Norden des Ausschnitts kann in der Rheinschlaufe bei Möhlin auch der grosse Grundwasserstrom in einem tieferliegenden Schotterbett des Rheins und die Situation der Saline Riburg gezeigt werden. In einer Karte mit Reliefgrundlage, die mit Bezug auf den Massstab direkt mit der darunter liegenden geologischen Karte verglichen werden kann, wurden die tektonischen Verhältnisse für die ganze Schweiz nach neueren Grundlagen aus dem Atlas der Schweiz kartiert (S. 16).
Eine ganze Serie von Karten zeigt die Veränderungen, welche die Schweizer Landschaft durch die verschiedenen Vergletscherungen erfahren hat. Aufgrund von neuesten grossmassstäblichen Unterlagen von R. Hantke und einer Tafel aus dem Atlas der Schweiz von H. Jäckli konnten je eine Darstellung der maximalen Vergletscherung in der Wirmeiszeit und eines Vorstoss- oder Rückzugstadiums realisiert werden. Zusammen mit 5 grossmassstäblichen Karten vermitteln sie ein eindrückliches Bild daruiber, wo und wie die Gletscher unsere Landschaft prägten. Das Ausmass der allmählichen Veränderungen wird am Beispiel Pfäffikersee deutlich. Dass sie auch heute noch anhalten, veranschaulichen die beiden nur 45 Jahre auseinanderliegenden Gletscherstände des Roseg- und Tschiervagletschers. 2 Kartenpaare illustrieren die Veränderungen, welche verschiedene Landschaften durch ihre Bewohner erfahren haben. Die Saarebene im Jahre 1845 und die Venogemündung vor 100 Jahren sind Rekonstruktionen nach alten kantonalen Aufnahmen. Sie können ebenfalls mit dem heutigen Zustand verglichen werden. Mit der gleichen Zielsetzung wurden die drei Städtekarten Zürich, La Chaux-de-Fonds und Genf bearbeitet, jedoch mit verschiedenen Varianen der Darstellungsform. Die Städte Bern und Basel andererseits werden in ihrer funktionalen Gliederung gezeigt. Bei Basel sind zusätzlich eine Reihe von Aspekten der Rheinschiffahrt eingetragen.

Aus kartentechnischer Sicht stellen fast alle neuen Karten überdurchschnittlich hohe Anforderungen. In vielen Fällen verlangt das feingliedrige Gefüge aller Kartenelemente ein iberlegtes Koordinieren und einen präzisen Passer. Die Reinzeichnungen von neuen Kartennetzen wie auch sämtliche Signaturenschichten der wirtschaftskarten wurden am Interaktiven GraphikSystem des Instituts für Kartographie der ETH Zürich entworfen und mit einer Präzisionszeichenmaschine gezeichnet. Trotz dieser spürbaren Entlastung des Arbeitsablaufs bleibt der reproduktionstechnische Aufbau der komplexen Wirtschaftskarten äusserst anspruchsvoll. In Anbetracht dieser besonderen Schwierigkeiten darf die kartographische, reprotechnische und drucktechnische Arbeit als vorzüglich bezeichnet werden.

\section{Ausblick auf die nächste Auflage}

Die Redaktion und die Beratende Kamission befassen sich gegenwärtig mit dem Sammeln und Sichten der Reaktionen auf die Zwischenauflage. Beide Stellen nehmen Fehlermeldungen und Kritik gerne entgegen. Willkammen sind natürlich auch positive Echos, damit vereinzelter Kritik nicht zu grosses Gewicht beigemessen wird. Ueber den Verein Schweizer Geographielehrer versuchten wir weitere Interessenten für gelegentliche Mitarbeit an den Redaktionsarbeiten zu gewinnen, bis jetzt allerdings nur mit beschränktem Erfolg. Zweifellos verfügen wir in unserem Lande über ein bedeutendes potential an geographischen 
Kenntnissen über fast sämtliche Räume der Erde. Unterstützung von dieser Seite beim Ausmerzen von Fehlern, beim Bearbeiten einzelner Karten oder bei der Durchsicht von Entwïrfen wären uns sehr willkonmen und könnten die Qualität dieses Kartenwerkes nur steigern.

Ein Inhaltsverzeichnis für die vollständig erneuerte Ausgabe liegt zwar bereits vor. Es wird nun, zusammen mit der Beratenden Komission, überprüft und verfeinert. Der Beratenden Kommission werden laufend Karten- und Themengruppen und Dispositionen zur Begutachtung vorgelegt. Wir sind auch bestrebt, die ersten Erfahrungen mit der Zwischenauflage für die nächste Auflage in den neuen Entwürfen zu berüiksichtigen. Der Absatz der Zwischenauflage hat inzwischen höchst erfreulich eingesetzt und das Bedürfnis nach einem Schweizer Schulatlas nachhaltig unterstrichen. Das ermutigt uns, die Arbeiten mit im wesentlichen unveränderter Zielsetzung weiterzuführen.

Prof. Ernst Spiess, Institut für Kartographie, ETH-Hönggerberg, 8093 Zürich

\section{Literaturbesprechung}

DIVERSE Autoren: Berichte und wissenschaftliche Abhandlungen des 42. Deutschen Geographentages (Juni 1979). 612 S., 82 Abb., Verlag Franz Steiner, Wiesbaden, 1980, DM 88.--

Mit dem Göttinger Treffen waren, wie der vorliegende Verhandlungsbericht besagt, einige Neuerungen: weniger Hauptvorträge, zahlreichere Fachsitzungen, verknüpft; die letzteren sind deshalb verkürzt publiziert, auch fehlen die Diskussionen. Beides ist bedauerlich.

Gleichwohl bietet der Band das weite und differenzierte Spektrum der Gesamtgeographie. In 11 "Hauptvorträgen" und 28 Fachsitzungen wurden etwa 170 Themen behandelt, die an dieser Stelle kaum zu würdigen sind. Die Hauptvorträge befassten sich mit der angewandten Geomorphologie ( $\mathrm{H}$. Mensching) und den "Klimarelieftypen" (J. Hagedorn), dem Klima der Erde (A. Kessler), den ländlichen Siedlungen (H. J. Nitz), der Stadtgeographie (E. Lichtenberger), den Industrieräumen (K. Hottes), der geographischen Fernerkundung (H.G. Gierloff, F. Wieneke), der Theoretischen Geographie (E. Wirth), "Zweitausend Jahre Geographie (J. Hövermann) sowie dem "Curriculum" (A. Schultze, H. Haubrich). In den Fachsitzungen widmeten sich verschiedene Gruppen den Problemen der Formkonvergenz, der Klimaformen und der geamorphologischen Kartierung, der Klimaschwankungen, der Trockengrenzen, der Modelle in der physischen und anthropischen Geographie (inkl. Raumplanung), der aussereuropäischen Kolonisation, der Stadtentwicklung, der periodischen Märkte, der Zentralitätsforschung, der sozialräumlichen Wirkungsanalyse, der Fortschritte der Industriegeographie, der Raumnutzung in Peripherräumen, der sozialräumlichen Entwicklungstheorien in geographischer Sicht, der Ideengeschichte der Geographie und der Organisation des Unterrichts in Volks- und Hochschulen. Drei Schweizer lieferten beachtliche Beiträge zu Fragen der Förderung von Regionalzentren (H. Elsasser) und zur Klimatologie des Schweizer Mittellandes (Chr. Pfister, H. Wanner). Im Ganzen zeigt sich eine erfreuliche Auffassung vom Wesen und von den vielfältigen Aufgaben der Geographie gegenüber einer gewissen Ernüchterung hinsichtlich der polemischen Aeusserungen der Vorjahre. Das geht auch aus den einleitenden Ansprachen hervor, die sowohl die Verbundenheit der deutschen und der Geographen überhaupt wie die ständig wachsenden Probleme betonen. Vor allem werden sie eindrücklich aus der Eröffnungsrede Prof. G. Sandners, der bekräftigt, Geographie sei "keine Wissenschaft in der Krise", sondern bei allen schwebenden Fragen und Schwierigkeiten, ein Forschungszweig, der unter bestimmtem "Auftrag und Forderung" stehe und dessen wichtigster Auftrag darin zu sehen sei, beizutragen zur Lösung der imer "schwerer ... werdenden Konflikte unserer Welt". Der ganze Bericht wird so zum überzeugenden Beweis für den Realisierungswillen dieser Absichten.

GH $3 / 82$ Ernst Winkler, Zürich 